\title{
Anti-nicastrin monoclonal antibodies elicit pleiotropic anti-tumour pharmacological effects in invasive breast cancer cells
}

\author{
Aleksandra Filipović • Ylenia Lombardo $\cdot$ Monica Fronato $\cdot$ Joel Abrahams \\ Eric Aboagye • Quang-De Nguyen • Barbara Borda d'Aqua • Anne Ridley • \\ Andrew Green · Emad Rahka - Ian Ellis · Chiara Recchi • Natasa Przulj • \\ Anida Sarajlić $\cdot$ Jean-Rene Alattia $\cdot$ Patrick Fraering $\cdot$ Mahendra Deonarain • \\ R. Charles Coombes
}

Received: 26 August 2014/Accepted: 27 August 2014/Published online: 24 September 2014

(C) The Author(s) 2014. This article is published with open access at Springerlink.com

\begin{abstract}
The goal of targeted cancer therapies is to specifically block oncogenic signalling, thus maximising efficacy, while reducing side-effects to patients. The gamma-secretase (GS) complex is an attractive therapeutic target in haematological malignancies and solid tumours with major pharmaceutical activity to identify optimal inhibitors. Within GS, nicastrin (NCSTN) offers an opportunity for therapeutic intervention using blocking monoclonal antibodies (mAbs). Here we explore the role of anti-nicastrin monoclonal antibodies, which we have developed as specific, multi-faceted inhibitors of proliferation and invasive traits of triple-negative breast cancer cells. We use 3D in vitro proliferation and invasion assays as well as an orthotopic and tail vail injection triple-negative breast cancer in vivo xenograft model systems. RNAScope assessed nicastrin in patient samples. AntiNCSTN mAb clone-2H6 demonstrated a superior anti-
\end{abstract}

Electronic supplementary material The online version of this article (doi:10.1007/s10549-014-3119-z) contains supplementary material, which is available to authorized users.

A. Filipović $(\square) \cdot$ Y. Lombardo $\cdot$ M. Fronato $\cdot$ J. Abrahams E. Aboagye - Q.-D. Nguyen - C. Recchi - R. C. Coombes Division of Surgery and Cancer, Department of Oncology, ICTEM Hammersmith Hospital Campus, Imperial College London, Du Cane Road, W12 0NN London, UK

e-mail: a.filipovic@imperial.ac.uk

B. B. d'Aqua · A. Ridley

Randall Division of Cell and Molecular Biophysics, King's

College London, SE1 1UL London, UK

\section{A. Green · E. Rahka · I. Ellis}

Department of Histopathology and School of Molecular Medical

Sciences, The University of Nottingham and Nottingham

University Hospitals NHS Trust, Nottingham City Hospital,

Nottingham, UK tumour efficacy than clone-10C11 and the RO4929097 small molecule GS inhibitor, acting by inhibiting GS enzymatic activity and Notch signalling in vitro and in vivo. Confirming clinical relevance of nicastrin as a target, we report evidence of increased NCSTN mRNA levels by RNA in situ hybridization (RNAScope) in a large cohort of oestrogen receptor negative breast cancers, conferring independent prognostic significance for disease-free survival, in multivariate analysis. We demonstrate here that targeting NCSTN using specific mAbs may represent a novel mode of treatment for invasive triple-negative breast cancer, for which there are few targeted therapeutic options. Furthermore, we propose that measuring NCSTN in patient samples using RNAScope technology may serve as companion diagnostic for anti-NCSTN therapy in the clinic.

Keywords Nicastrin $\cdot$ Breast cancer $\cdot$ Monoclonal antibodies

N. Przulj · A. Sarajlić

Department of Computing, Imperial College London, London, UK

J.-R. Alattia $\cdot$ P. Fraering

Brain Mind Institute, School of Life Sciences, Ecole Polytechnique Federale de Lausanne (EPFL), 1015 Lausanne, Switzerland

M. Deonarain

Department of Life Sciences, Imperial College London, Exhibition Road, SW7 2AZ London, UK 


\section{Introduction}

The vision of selective targeting was introduced a century ago by Paul Ehrlich who hypothesised that the 'magic bullet' could be developed to combat malignant disease. Patient-tailored treatment entails taking into account the molecular makeup of an individual tumour in order to target the cancer-driving pathways and ensure a maximal antitumour response. In this regard, introduction of monoclonal antibodies (mAbs) as therapies targeted against a specific oncogenic molecule expressed in cancer tissue, is one of the most significant recent advances in the treatment of leukaemias and solid tumours. Case in point is Trastuzumab, a $\mathrm{mAb}$ targeting the HER2 receptor, which confers significant survival benefit to a subset of HER2 overexpressing/amplified, and particularly aggressive breast cancers.

To this day, however, treatment of oestrogen receptor (ER)-/progesterone receptor (PR)-/HER2- negative (triple negative) breast cancer (TNBC) represents a challenge, given the absence of well-defined molecular targets. TNBCs are biologically more aggressive, and chemotherapy remains the mainstay treatment option in the neoadjuvant, adjuvant and metastatic setting. Therefore, there is a clear unmet medical need to better understand the molecular basis of TNBC and to develop effective targeted treatments. Dependence of TNBC cells on Notch activity has been recently demonstrated not only in metastatic disease, but also in primary tumours where Notch 1 and Notch 4 are overexpressed and localised in the cytoplasm and the nucleus, indicating their activated state and conferring worse overall survival (Reedijk et al., Can Res 2005; Speiser et al., Int J Surg Path 2011). Importantly, a subset of these breast cancers are driven by a Notch fusion gene, which renders the cells addicted to the Notch pathway for survival. Notch receptors are activated by the gammasecretase (GS) enzyme, which is increasingly gaining recognition as a relevant target in human malignancies. Gammasecretase is composed of Presenilin, Aph-1, Presenilin enhancer protein (PEN-2) and Nicastrin (NCSTN). Gammasecretase inhibitors (GSIs) are small molecules that inhibit the catalytic activity of Presenilin within the GS, thereby being responsible for the unselective inhibition of the wide range of GS substrates (Her4, CD44, E-cadherin, N-cadherin, MUC-1, EPCAM etc.), in addition to Notch receptors. Nicastrin (NCSTN) is the key component of GS, as it enables its composition, maintains enzyme stability and executes GS-substrate recognition (De Strooper Cell 2005). Recently, a gamma-secretase independent function of NCSTN has been proposed, in the process of controlling p53-mediated cell death via the Akt pathway. Nicastrin is also the only component of the GS with a single-pass trans-membrane domain and a large extracellular domain containing functional regions. We previously validated NCSTN as a target in breast cancer by demonstrating that it is overexpressed in breast cancer tissue compared to normal breast, where its abundance confers worse overall survival in the ER-negative cohort. Furthermore, we have shown that NCSTN is involved in sustaining the invasive phenotype in TNBC, while its genetic depletion using siRNA and shRNA is sufficient to inhibit tomour growth in vitro and in vivo. We then introduced the concept of targeting NCSTN using antagonistic antibodies [1], which has since been followed up in mouse models of T-ALL [2]. Using genetic immunisation, we have developed mAbs against NCSTN extracellular domain, as a modality to block NCSTNdriven signalling in invasive breast cancer cells. The major findings of our study centre on the anti-tumour and anti-metastatic effects obtained by targeting NCSTN using our $\mathrm{mAbs}$ in TNBC, in vitro and in vivo. Finally, we propose that assessing NCSTN mRNA expression status in patient tissue, using a novel, in situ RNA hybridization technology (RNAScope), may be exploited in the clinic for tailored patient stratification and monitoring of treatment efficacy.

\section{Results}

We raised anti-NCSTN monoclonal antibodies (mAbs) by genetic immunisation using cDNA, encoding for the extracellular domain of NCSTN (34-669 a.a.) (Supplementary Fig. 1a). A dual discrimination approach was used to identify only those clones that recognised endogenous NCSTN on the surface of breast cancer cells, and inhibited their invasion capacity. Of the $21 \mathrm{mAb}$ clones screened by FACS and the transwell invasion assay, ten clones $(1 \mathrm{E} 2,1 \mathrm{H} 4,2 \mathrm{H} 6,6 \mathrm{H} 5$, $6 \mathrm{H} 11,9 \mathrm{G} 7,9 \mathrm{~F} 1,10 \mathrm{C} 5,10 \mathrm{C} 11,10 \mathrm{E} 9)$ recognised endogenous NCSTN on non-permeabilized MDA-MB-231 cells and of those seven emerged as candidates from the transwell invasion assay $(1 \mathrm{H} 4(44 \pm 12.4 \%), 2 \mathrm{H} 6(60 \pm 5.6 \%), 6 \mathrm{H} 5$ $(39 \pm 2.6 \%), 6 \mathrm{H} 11(40 \pm 6.4 \%), 9 \mathrm{G} 7(42 \pm 11.2 \%)$, $10 \mathrm{C} 5(65 \pm 10.2 \%)$ and $10 \mathrm{C} 11(50 \pm 10.3 \%)$, while three clones (1E2, 9F1 and 10E9) had no functional effect (Supplementary Fig. 1b, c).

We identified mAb clone-1E2 was to serve as a "bindingonly control' since it had no effect on invasion despite recognising cell surface NCSTN by FACS, while $2 \mathrm{H} 6$ and $10 \mathrm{C} 11$ were pursued as lead candidates. Their binding to endogenous NCSTN was confirmed in other TNBC cell lines (BT549, HCC1806) (Fig. 1a), where it was superior compared to binding to surface NCSTN on a luminal A breast cancer cell line (T47D). The binding of whole IgGs was measured directly by ELISA. The antigen used was the mammalian NCSTN ECD-Fc fusion protein (Fusion Antibodies). Surface plasmon resonance was used to epitope map the anti-NCSTN mAbs by determining which ones compete for binding to the NCSTN antigen. An indicated $\mathrm{mAb}$ was immobilized onto the surface of the BIACore chip, which was then exposed to the flow of the human NCSTN- 
a
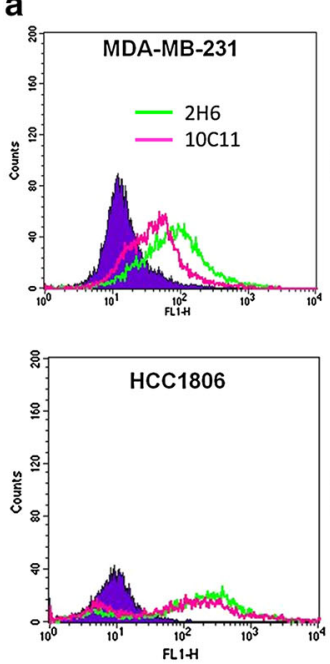

b
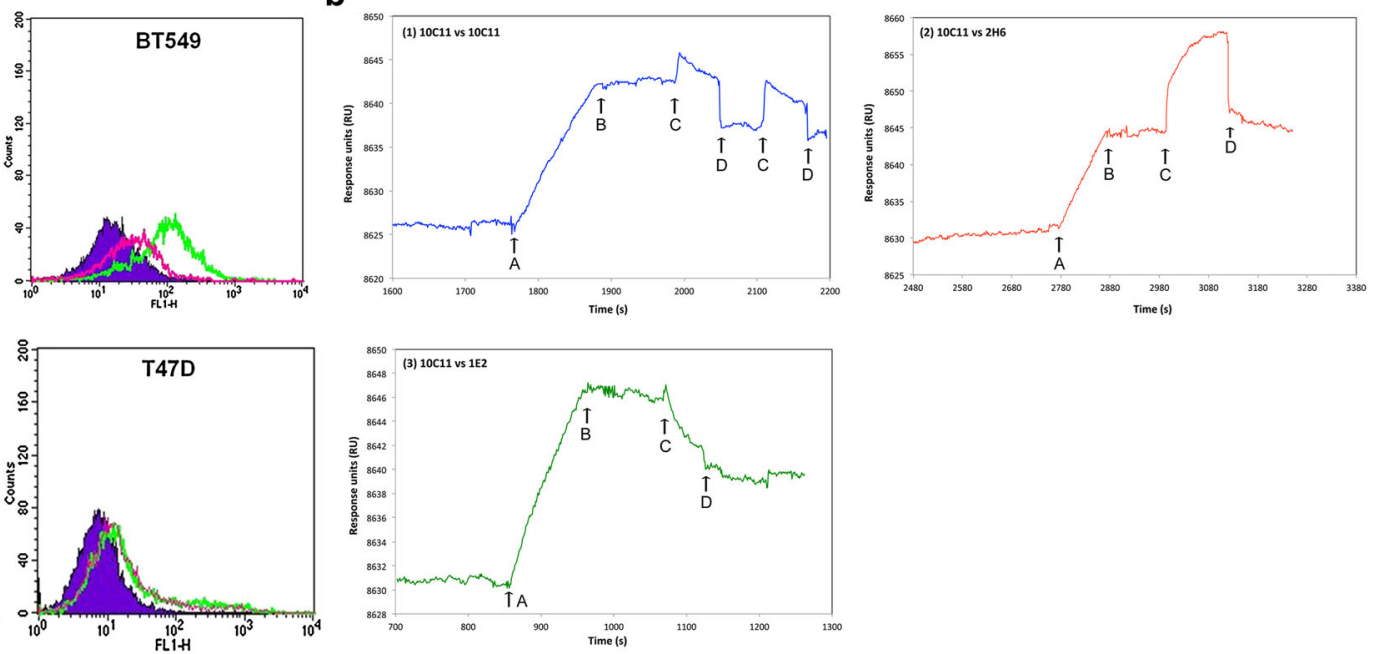

c
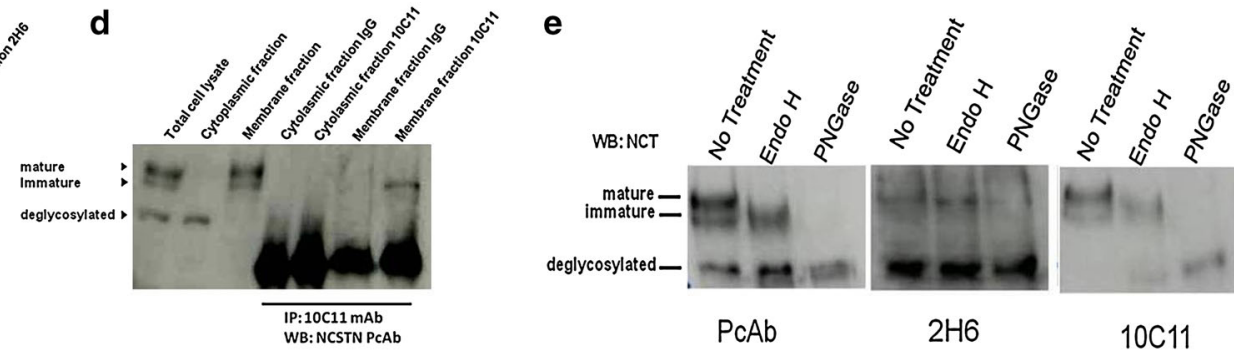

Fig. 1 Binding properties of anti-nicastrin monoclonal antibodies in cell-based and non-cell-based assays. a Non-permeabilized MDAMB-231, BT549, HCC1806 and T47D cells were incubated with the Rat IgG2b isotype control (blue), 2H6 (green) and 10C11 (pink) at $50 \mu \mathrm{g} / \mathrm{ml}$. Anti-Rat-FITC conjugate was used as a secondary antibody. Histograms indicate a shift upon binding of the antiNCSTN mAbs to NCSTN on cancer cells. b BIACore binding competition. Monoclonal antibody clone 10C11 was immobilised directly onto a CM5 chip and the antigen, NCSTN-Fc was flowed over at $1 \mathrm{mM}$ concentration. NCSTN binding 'start' and 'end' is marked with 'A' and 'B', respectively. After a short period to allow for any weak dissociation, the test monoclonal antibodies (1) 10C11, (2) $2 \mathrm{H} 6$, and (3) $1 \mathrm{E} 2$ were flowed over as Fab fragments at $1 \mathrm{mM}$ concentration. The start and ends are marked with ' $\mathrm{C}$ ' and ' $\mathrm{D}$ ', respectively. Additive binding (indicating a separate, non-overlapping epitope) is seen as a further increase in the sensogram signal, whereas competitive binding is seen as a dissociation curve. Clone $2 \mathrm{H} 6$ is shown to bind additively with clone $10 \mathrm{C} 11$. c, d Cell fractionation was performed using the ProteoExtract ${ }^{\circledR}$ Transmembrane Protein Extraction Kit (Novagen). Immunoprecipitation was done with $2 \mathrm{H} 6$

Fc protein. The sensogram registered a consequent increase in the chip weight corresponding to the association part of the curve. Next, a chosen Fab fragment was injected and flown over the chip surface. We observed that 10C11 and $1 \mathrm{E} 2$ clones are binding overlapping epitopes, while 10C11 and $2 \mathrm{H} 6$ clones are directed against spatially distinct regions on the NCSTN ECD (Fig. 1b). The mAbs displayed moderate to high affinities on immobilised NCSTN ECD with $K_{\mathrm{d}}$ values of $0.61 \mathrm{nM}$ (10C11), $3.7 \mathrm{nM}$ (1E2) and $21.6 \mathrm{nM}$ (2H6). Cell-based quantitative FACS on non-permeabilized

and $10 \mathrm{C} 11 \mathrm{mAbs}$, and Western blots were probed using the nicastrin polyclonal antibody developed at Biogenes, GE against the nicastrin extracellular domain protein. e Nicastrin deglycosylation using EndoH and PNGase from whole cell lysates of MDA-MB-231 cells. Upon complete NCSTN deglycosylation with PNGase, 1E2, 2H6, $10 \mathrm{C} 5$ and 10C11 mAbs recognise the NCSTN non-glycosylated core protein at $80 \mathrm{kDa}$. This suggests that the mAbs bind peptide regions on the NCSTN ECD. Cell fractionation experiment showing that the $10 \mathrm{C} 11 \mathrm{mAb}$ is most specific for membrane bound Nicastrin. Immunopreciptation of NCSTN from both the cytoplasmic (cyto) and the membrane (memb) fractions of MDA-MB-231 cells using $10 \mathrm{C} 11$ and $2 \mathrm{H} 6 \mathrm{MAb}$ confirms that these mAbs binds membrane NCSTN. e Western blotting and glycosidase treatments of nicastrin in cellular extracts of MDA-MB-231 cells are shown. EndoH and PNGase treatments show the sensitivity of the various bands to deglycosylation. Native, fully deglycosylated nicastrin migrates at $\sim 80 \mathrm{kDa}$, which corresponds to the predicted molecular weight for the native protein. Treatment with PNGase produced a deglycosylated form of nicastrin detected by mAbs

MDA-MB-231 cells established avidities of all three clones (Table 1; Supplementary Fig. 2). As well as being physiologically relevant, these cellular $K_{\mathrm{d}}$ values suggested predominantly monovalent binding or other GS complexrelated steric factors involved. Since functional, purified GS from mammalian cells contains predominantly mature, membrane-anchored NCSTN [3], it is of importance that $2 \mathrm{H} 6$ and $10 \mathrm{C} 11$ were particularly effective in recognising and immunoprecipitating NCSTN from the cellular membrane fraction containing the active protein [4] (Fig. 1c, d). 
a
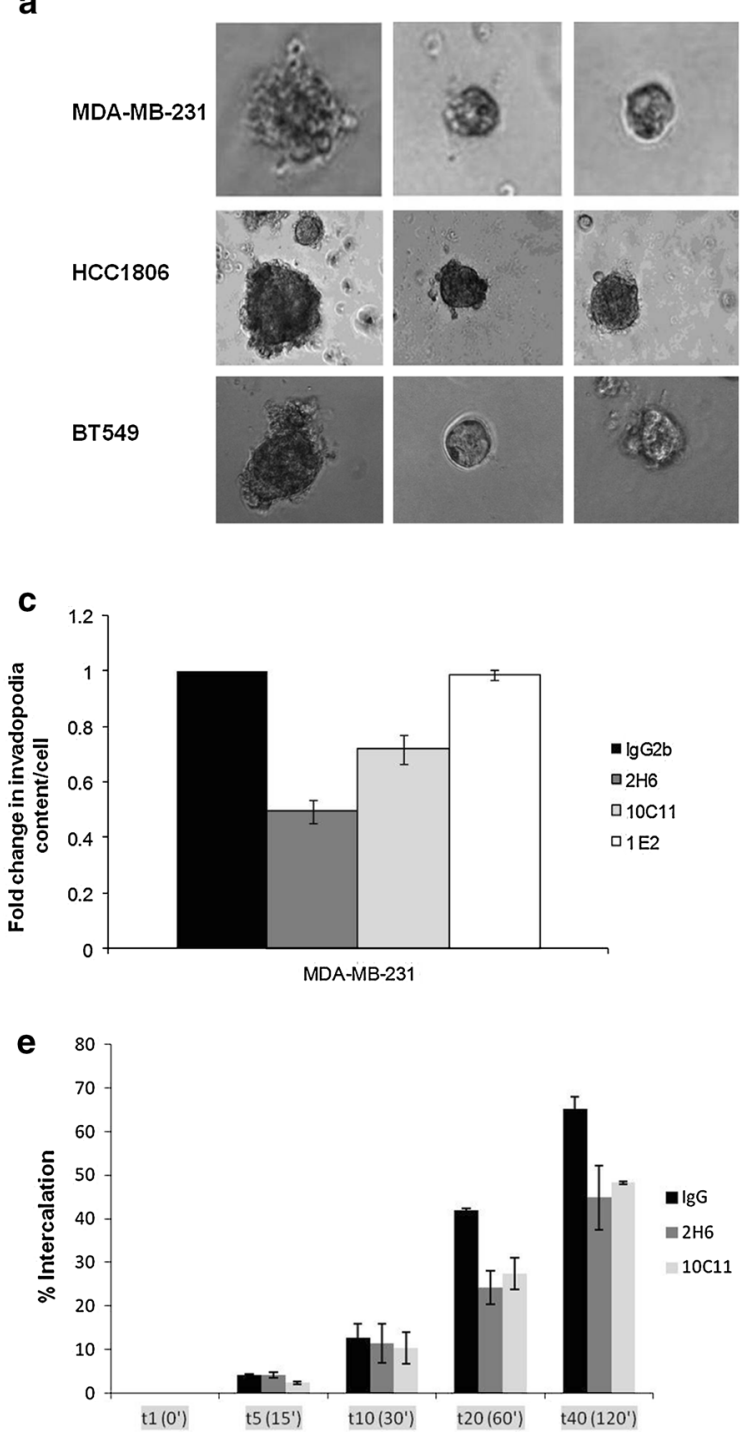

Fig. 2 In vitro functional effects of anti-nicastrin monoclonal antibodies on breast cancer cells. a Treatment of triple negative breast cancer cells with anti-NCSTN mAbs inhibits growth of acinilike structures. MDA-MB-231, BT549, HCC1806 cells $\left(1 \times 10^{3} \%\right.$ chamber) were plated in the 3D Matrigel overlay experiment. Cells were cultured in the media containing $2 \%$ Matrigel, supplemented with the isotype control Rat IgG antibody and the $2 \mathrm{H} 6$ and $10 \mathrm{C} 11$ $\mathrm{mAbs}$ at $100 \mu \mathrm{g} / \mathrm{ml}$. Treatment was added upon plating and every $48 \mathrm{~h}$ until Day 6. Cells were then imaged using the phase contrast microscopy. Magnification $\times 20$ b MDA-MB-231 cells were treated with indicated anti-NCSTN mAbs and subjected to the transwell invasion assay for $72 \mathrm{~h}$. Bars represent mean number of invaded cells \pm SEM from three separate experiments. Statistical difference from the respective concentration of the $\mathrm{IgG} 2 \mathrm{~b}$ isotype control (2H6 $50 \mu \mathrm{g} / \mathrm{ml}, p=0.005 ; 2 \mathrm{H6} 200 \mu \mathrm{g} / \mathrm{ml}, p=4.95 \mathrm{E}^{-05} ; 10 \mathrm{C} 1150 \mu \mathrm{g} / \mathrm{ml}$, $p=0.0007 ; 10 \mathrm{C} 11200 \mu \mathrm{g} / \mathrm{ml}, p=0.0002)$. c Gelatin degradation assay. MDA-MB-231 cells were treated with anti-NCSTN mAbs 2H6 and $10 \mathrm{C} 11$ at $100 \mu \mathrm{g} / \mathrm{ml}$ for $72 \mathrm{~h}$, trypsinyzed and re-plated onto FITC-Gelatin/Poly-Lysine coated glass slides where they were incubated for $7 \mathrm{~h}$ and analysed for formation of invadopodia. Bars represent fold change of gelatine degradation/cell in each treatment
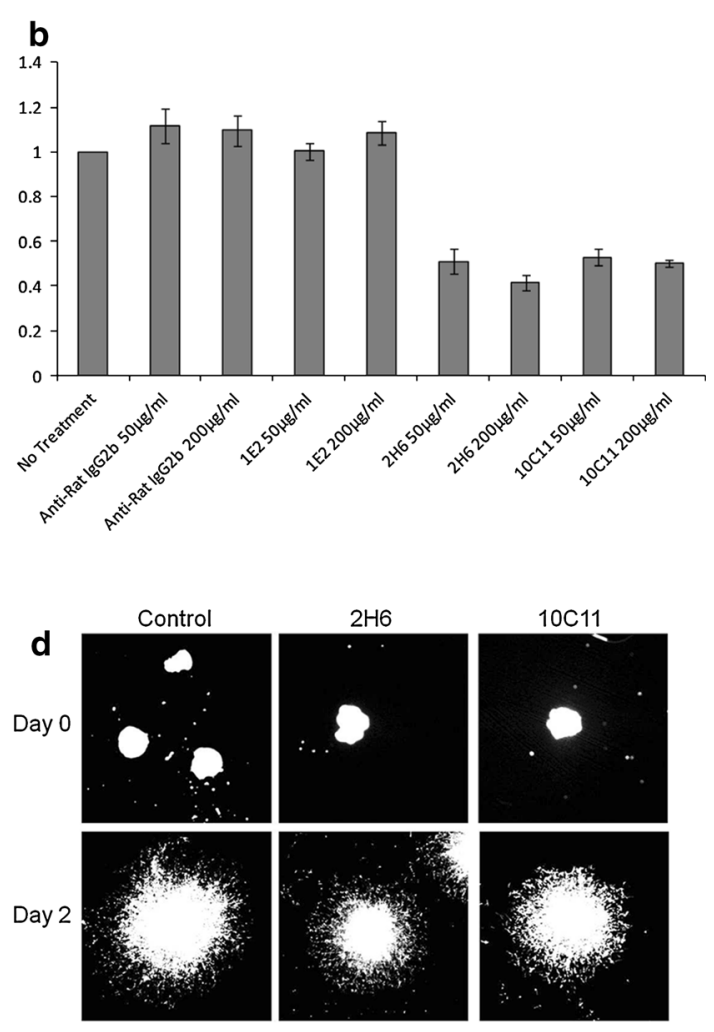

f

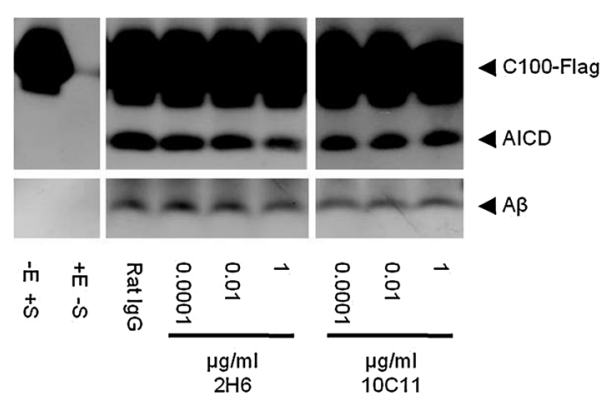

condition \pm SEM from three separate experiments. Calculations were done based on randomly assessing degree of gelatine degradation/cell in at least $n=100$ cells per treatment condition. d MDA-MB-231 were treated with $2 \mathrm{H} 6$, and $10 \mathrm{C} 11 \mathrm{mAbs}$ at $100 \mu \mathrm{g} / \mathrm{ml}$ and allowed to form cell spheroids, generated by hanging drop culture, which were then embedded in polymerised Collagen I. Images reveal the spheroids at Day 0 and Day 2, at which point cell penetration of the collagen I matrix was apparent. Spheroids were fixed and stained by actin staining with AlexaFluor488-Phalloidin, and entire spheroids were visualised at magnification $\times 2.5$. e Anti-NCSTN mAbs inhibit cancer cell intercalation between endothelial cells. Graphs show percent of intercalated cancer cells at the designated time point annotated. Cancer cells were filmed on HUVECs for $120 \mathrm{~min}$. In each experiment, $\geq 100$ cells were analysed in at least three fields. Data are expressed as mean percentage intercalated cells. Statistical difference from the IgG2b isotype control (2H6 $5060^{\prime}, p=0.0006 ; 2 \mathrm{H} 6120^{\prime}$, $\left.p=007 ; 10 \mathrm{C} 1160^{\prime}, p=0.001 ; 10 \mathrm{C} 11120^{\prime}, p=0.01\right)$. f Cell-free gamma secretase assay of purified gamma secretase which was preincubated for $4 \mathrm{~h}$ with $2 \mathrm{H} 6$ and $10 \mathrm{C} 11 \mathrm{mAbs}$ prior to activity which was assayed by detection of beta amyloid cleavage and detection of the AICD cleavage product by Western blotting 
Since NCSTN is heavily glycosylated and dependent on this post-translational modification for maturation and function, we further explored the ability of our antibodies to recognise NCSTN glycoforms by using EndoH and PNGase. EndoH and PNGase treatment to remove the post-translationally added glycans revealed that $2 \mathrm{H} 6$ and $10 \mathrm{C} 11$ recognised the non-glycosylated protein at $\sim 80 \mathrm{kDa}$ (Fig. 1e) [4], indicating that the mAb-binding epitopes are likely to represent peptide regions with conformation not entirely dependent on maturation through glycosylation.

Next, we assessed therapeutic potency of anti-NCSTN mAbs in a variety of in vitro, three-dimensional, Matrigel/ Collagen I-based assays, allowing for incorporation of the physiologically relevant, tumour cell-extracellular matrix interactions [5]. In such conditions, both purified 2H6 and 10C11 mAbs potently inhibited Matrigel acini formation in a panel of TNBC cell lines (MDA-MB-231, BT549, HCC1806) (Fig. 2a). Moreover, grape-like acini converted to rounded structures upon $\mathrm{mAb}$ treatment, indicating that the invasive capacity of the cells was being attenuated [6], which was confirmed in a designated Boyden chamber assay (Fig. 2b). Clone 2H6 was superior in attenuating gelatin degradation by cellular invadopodia protrusions (Fig. 2c), as well as the invasive capacity of MDA-MB-231 spheroids into Collagen I (Fig. 2d), with an average increase of sphere volume from Day 0 to Day 2 of 20.1-fold for the Control IgG and 16.8-fold for the 2H6-treated spheres $(p=0.03)$. To test whether NCSTN antibodies might affect the extravasation step of metastasis [7], we followed the interaction of MDAMB-231 cells with endothelial cells by time-lapse microscopy [8]. Treatment of MDA-MB-231 cells with $2 \mathrm{H6}$ and $10 \mathrm{C} 11$ did not affect the initial contact and adhesion between cancer cells and endothelial cells: however, it delayed the intercalation of MDA-MB-231 cells within the endothelial monolayer after 1 and $2 \mathrm{~h}$ of interaction (Fig. 2e). Moreover, only $2 \mathrm{H} 6$ inhibited the enzyme activity of the purified GS by $>50 \%$ (Fig. 2f) [9].

In vivo effects of the anti-NCSTN mAbs were evaluated in two independent xenograft models. First, in an orthotopic model of breast cancer using luciferase labelled MDA-MB231 cells implanted into mammary fat pads of nude mice, $2 \mathrm{H} 6$ antibody inhibited tumour growth by $50 \%(p=0.007)$ with concomitant $\mathrm{Ki} 67$ reduction, compared to the $35 \%$ inhibition by 10C11 and negligible effects using RO4929097 GSI (Fig. 3a, b). Key to our premise that anti-NCSTN therapy may be distinct from small molecule GS inhibition, mice showed no histological evidence of Goblet cell hyperplasia in the antibody treated arms, while these effects were detectable in the GSI-treated animals [10] (Fig. 3c). Analysis of the excised tumours showed that $2 \mathrm{H} 6$ and 10C11 inhibited Notch activity as assessed by Notch target genes (Hes l, Heyl), as well as key molecular regulators of cancer cell invasion and tumour-stroma cross-talk (MMP7, MMP9, CD147 and Aktl/
2, p100 $\alpha$-catalytic (110 kDa) subunit of the PI3-kinase) [11, 12] (Fig. 3d, e). Secondly, in a metastatic xenograft model generated by tail vein inoculation of luciferase labelled MDA-MB-231 cells [13], a greater than $70 \%$ reduction $(2 \mathrm{H} 6$ vs. $\mathrm{IgG}, p=0.0006$; $2 \mathrm{H6}$ vs. PBS, $p=0.0027$ ) in metastatic growth was seen in the 2H6-treated animals (Fig. 4a-e, Supplementary Fig. 3 a-e4). Proliferative index Ki67 was reduced in metastatic deposits of 2H6-treated mice only (Fig. 4f), and we noted concomitant attenuation of Notch target genes (Hesl, Heyl, Hey2, HeyL), as well as key mediators of metastasis, such as periostin, MMP7, MMP9 [14, 15] (Fig. 4g, h). Safety of the antibody treatment was further confirmed by undisturbed body weight, liver and kidney function tests (Supplementary Fig. 4). The absence of side-effects could be readily interpreted as our mAbs recognise the murine NCSTN orthologue (Supplementary Fig. 4).

Having demonstrated that anti-NCSTN mAb therapy may be efficient in TNBC, we next attempted to identify the optimal co-diagnostic approach, with potential clinical translational utility. NCSTN validity as a target comes from its overexpression in breast cancer, as well as gene amplification in a subset of breast cancers, correlating with adverse overall survival [1] (Supplementary Fig. 5 a, b). Analysis of the Nature TCGA cohort [16] revealed a subset of genes $(n=21)$ (Supplementary Table 1,2$)$ co-amplified and concomitantly mutated $(n=2)$ only in NCSTNamplified cases, proposing potential novel molecular determinants contributing towards oncogenic signalling in selected breast cancer cases. To complement our own immunohistochemistry [1] and the Nature TCGA 2012/cBioPortal amplification-data, we have utilised a novel RNA in situ hybridization platform (RNAScope; Advanced Cell Diagnostics, Hayward, CA) [17], in a large oestrogen receptor alpha negative (ER $\alpha$-ve) cohort of breast cancer patients $(n>300)$. This allowed single-cell visualisation of NCSTN mRNA transcript levels and establishment of a corresponding $\mathrm{H}$-score as described in the supplementary methods. Median H-score of 120 was used as cut-off for statistical correlations with survival and patient/tumour characteristics. This demonstrated that high NCSTN RNAScope score of $\geq 120$ (0-400), strongly predicted worse disease-free survival ( $p=0.019$ ) over a 10-year follow-up (Supplementary Fig. 5 c, d), and represented an independent prognostic marker in multivariate analysis with tumour grade, stage and size ( $p=0.044)$ (Supplementary Table 3). Importantly, it positively correlated with NCSTN membrane expression assessed by immunohistochemistry, high Ki67 proliferative index, pleomorphism, mitotic rate of cancer cells, as well as aberrant cytoplasmic BRCA1 expression (Supplementary Table 4). Given that the NCSTN RNAScope was assessed in a pre-selected ER $\alpha$-ve cohort, where $>95 \%$ of tumours were of grade 3 and size above $1.5 \mathrm{~cm}$, these correlations indicate that high levels of NCSTN 


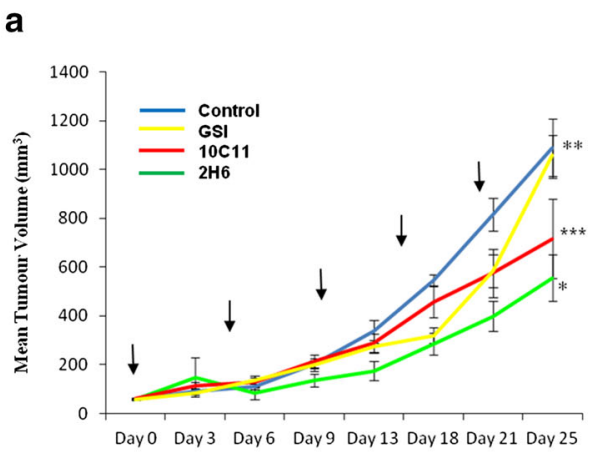

b

Ki67
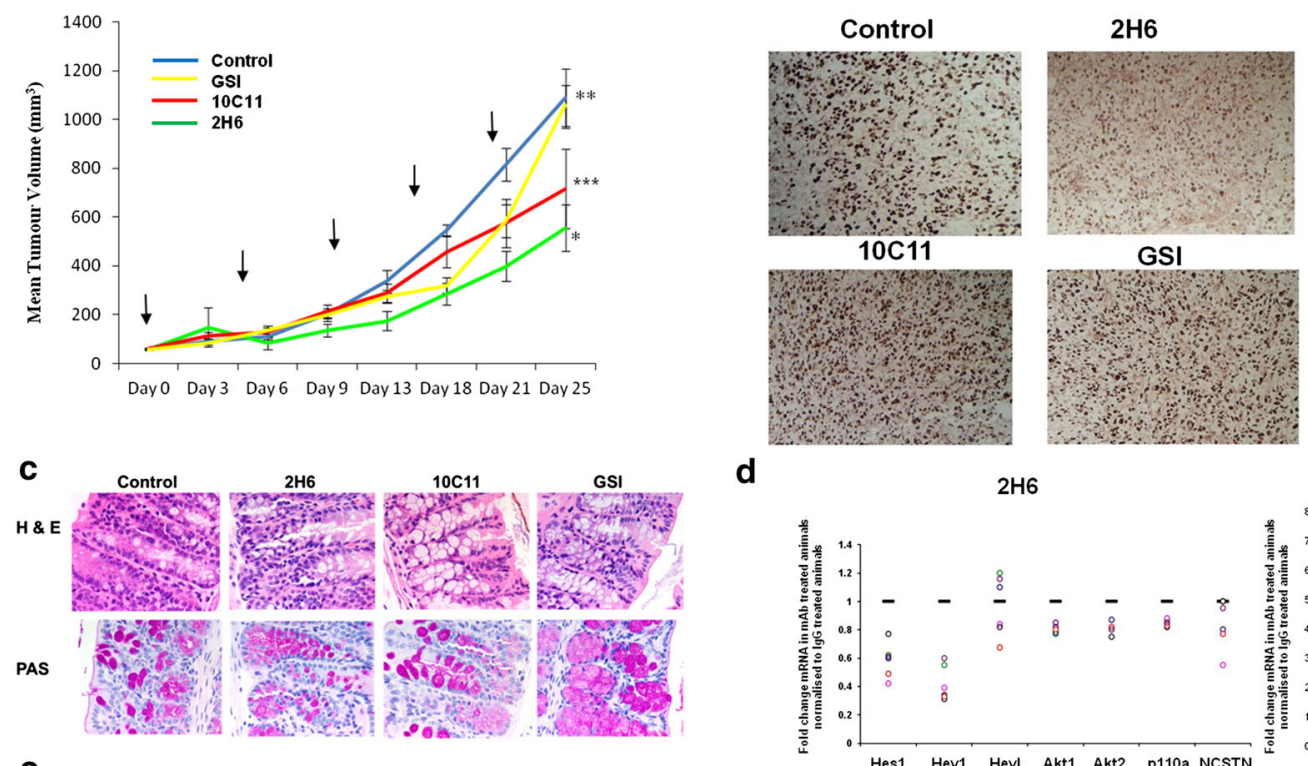

d
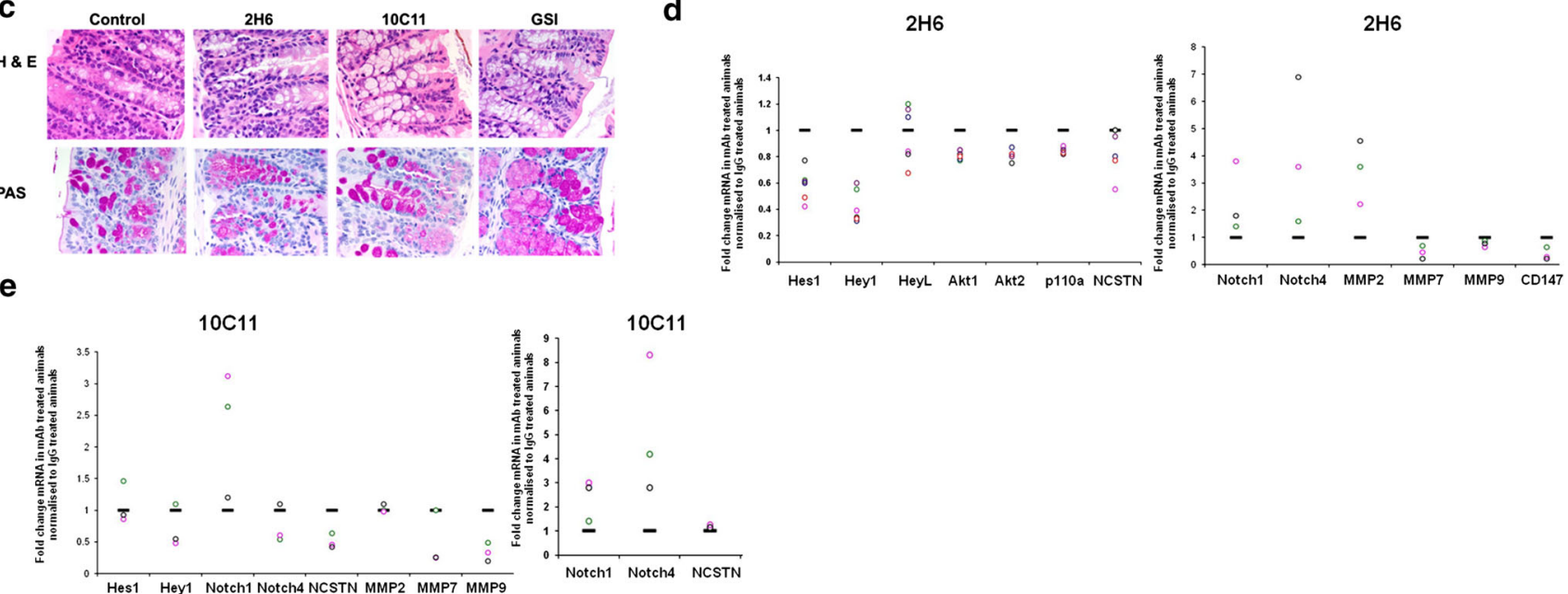

Fig. 3 In vivo effects of anti-nicastrin monoclonal antibodies in the orthotopic mouse model of triple-negative breast cancer. a-e In vivo model of triple-negative breast cancer MDA-MB-231 cells. MDAMB-231-luc-D3H2LN cells were injected into mammary fat pads of BalbC nude mice, and tumours were allowed to grow to $100 \mathrm{~mm}^{3}$. Animals were randomly stratified into control and treatment $(2 \mathrm{H} 6$, $10 \mathrm{C} 11$ and RO4929097) groups. Animals were treated by i.v. injection of $2 \mathrm{H} 6$ and $10 \mathrm{C} 11$ at $50 \mathrm{mg} / \mathrm{kg}$ every 5 days (total of 5 doses), and GSI was administered by oral gavage daily at $10 \mathrm{mg} / \mathrm{kg}$. Tumours were measured every 4 days using callipers. Mean tumour

mRNA transcript contribute to the aggressive nature of the $\mathrm{ER} \alpha$-ve disease.

\section{Discussion}

Our current study uses cellular, pre-clinical assays to demonstrate the importance of anti-NCSTN mAbs as multi-faceted therapeutics for TNBC.

The only major type of breast cancer for which no specific approved targeted therapy is available to improve patient outcome, is the triple negative subtype (Lehmann et al., JCI 2011). For this reason, it is essential that we identify molecules that are driving development and progression of TNBC. Gammasecretase enzyme can currently be targeted by gamma-secretase inhibitors (GSIs) (RO4929790, PF-03084014, MK-0752,

volumes \pm S.E.M. are plotted. Statistical Analysis: The data was statistically analysed using the Statistical Package for Social Sciences (SPSS)/15.0. For examining the improvement in the dependent variables on days 13-25 using two-way Repeated ANOVA was used. f Haematoxilin Eosin and Ki67 immunohistochemistry staining of excised and paraffin embedded. $\mathbf{g}, \mathbf{h}$ RT-qPCR analyses of genes from excised mammary fat pad tumours. Fold change of relative mRNA levels in treated animals compared to control is represented. Normalisation was done to GAPDH. Each coloured circle corresponds to an individual animal

BMS-906024, BMS-986115), which are in multiple clinical trials in haematological malignancies and advanced/metastatic/refractory solid tumours (Arcaroli et al., Drugs of the Future 2012). In breast cancer cells, single-agent GS inhibition does not greatly affect cell proliferation in vitro and in vivo, and metastatic models are lacking. Furthermore, rare cell lines and patient tumours with a Notch1 fusion gene, resulting in constitutively active Notch1, are most receptive to single-agent GSI growth inhibition (1), encouraging efforts to identify additional modalities to inhibit GS in cancer. Furthermore, true patient stratification methods lack for gamma-secretase inhibitor administration, as gamma-secretase activity levels or the expression levels of the gamma-secretase inhibitors' target, Presenilin, in cancer tissues, are not being evaluated. An optimal target for monoclonal antibody (mAb) therapy in cancer is a molecule expressed on the cell surface of the tumour cells, 

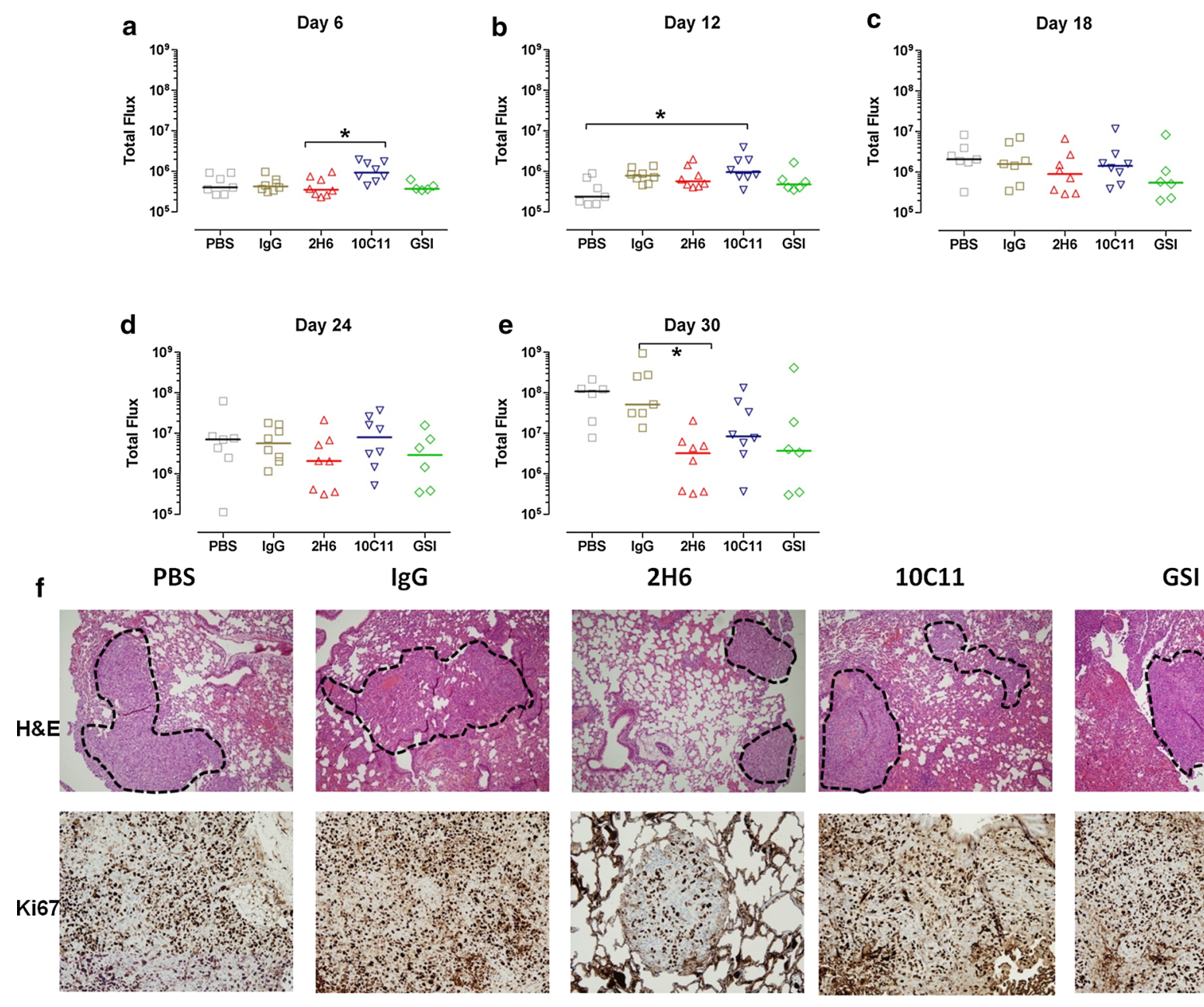

Day 24
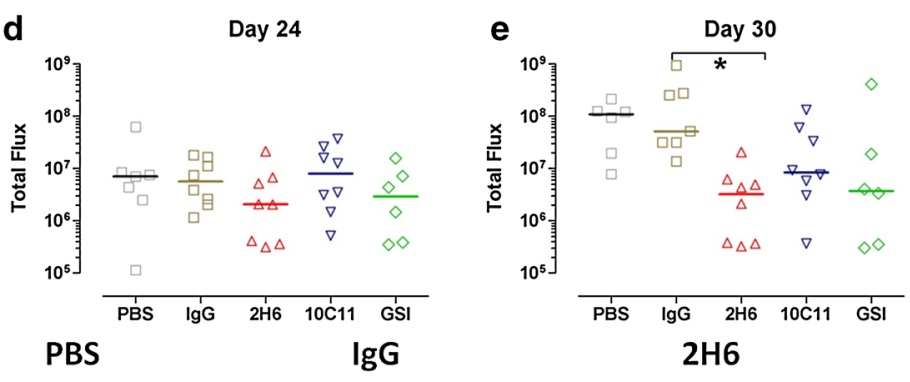

$10 C 11$
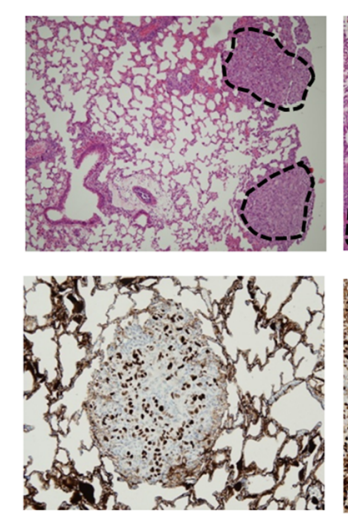

$\mathrm{h}$

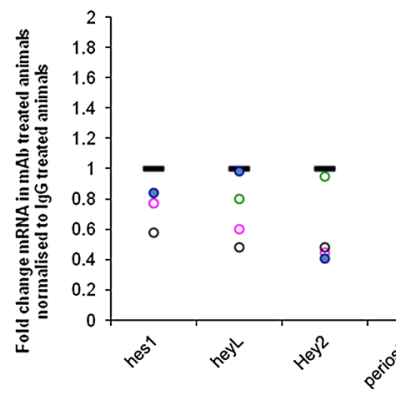

$10 \mathrm{C} 11$
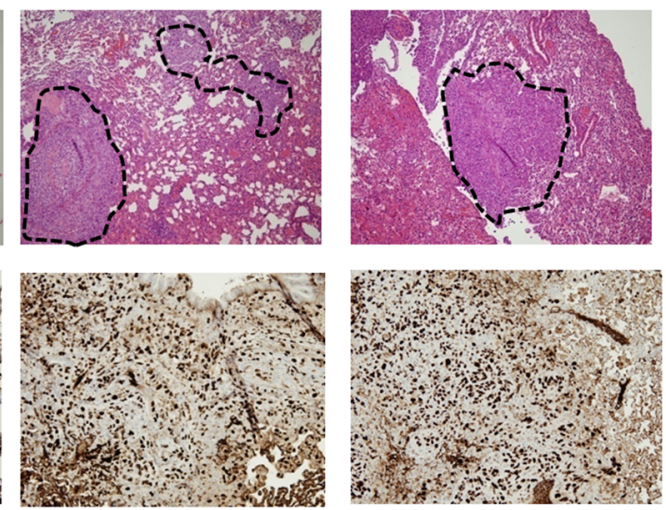

g

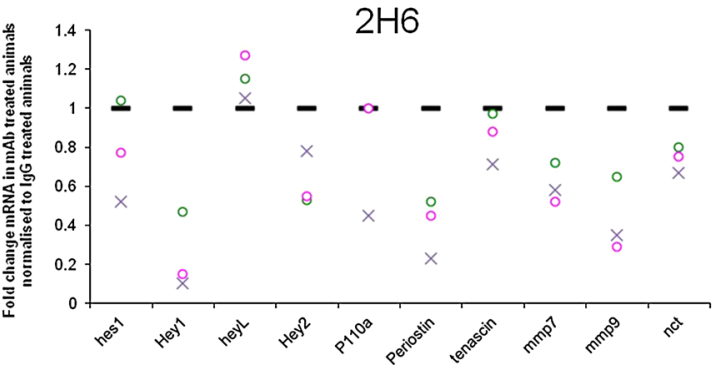

Fig. 4 In vivo effects of anti-nicastrin monoclonal antibodies in the metastatic mouse model of triple-negative breast cancer. a-e MDAMB-231-luc-D3H2LN $\left(1 \times 10^{6}\right)$ were injected into tail veins of BalbC nude mice, and treatment was initiated $72 \mathrm{~h}$ post cell inoculation. Anti-NCSTN monoclonal antibodies (2H6 and 10C11) and control rat $\mathrm{IgG}$ were administered i.v. at $50 \mathrm{mg} / \mathrm{kg}$ in 5 -daily intervals, while the RO4929097 was given by oral gavage at $10 \mathrm{mg} /$ $\mathrm{kg} / \mathrm{day}$. Mice were imaged weekly from dorsal and ventral views for 5 weeks to monitor development of metastasis. Bioluminescent imaging was performed with a highly sensitive, cooled CCD camera mounted in a light-tight specimen box (IVIS ${ }^{\mathrm{TM}}$; Xenogen). Imaging and quantification of signals were controlled by the acquisition and

whose expression and function contribute to the development and/or progression of the disease, and the inhibition of which selectively targets the cancer cells while inducing minimal analysis software Living Image ${ }^{\circledR}$ (Xenogen). Relative Luminescent Units were representative of tumour burden. All groups were compared to one another for each time point by 1-Way ANOVA (Kruskall-Wallis) and Dunn's Multiple Comparison Test to compare individual groups. Total luciferase flux is expressed as a log scale. $* p<0.05$. f Haematoxilin \& Eosin and Ki67 immunohistochemistry staining of excised and paraffin embedded-lung tissues. Black dotted lines demark metastatic tumour deposits. Ki67 staining is visualised as nuclear. g, h RT-qPCR analyses of genes from excised lung metastasis. Fold change of relative mRNA levels in treated animals compared to control is represented. Normalisation was done to $G A P D H$. Each coloured circle corresponds to an individual animal

side-effects. We have demonstrated that Nicastrin is a candidate therapeutic target in invasive breast cancer, druggable using a targeted blocking antibody. 
Our current study uses cellular and pre-clinical assays to demonstrate the importance of anti-NCSTN mAbs as multifaceted therapeutics for TNBC. Treatment with anti-NCSTN mAbs impinged not only on cancer cell proliferation, but also on the multiple, crucial steps of the metastatic cascade: (i) the invasive potential, (ii) ability of cancer cells to degrade ECM via invadopodia extension, as well as (iii) trans-endothelial extravasation efficiency, were all inhibited. Importantly, this translated into attenuation of primary tumour and metastatic growth in vivo. Previous work established that ant-NCSTN mAbs which bind the proximal part of the ECD (41-163 a.a.) fail to inhibit GS activity. Equally, anti-NCSTN mAb A5226A which binds to Arg391 fails to recognise murine orthologues, hindering in vivo toxicity assessment. Our BIACore and functional data propose that $2 \mathrm{H} 6$ and 10C11, which have differential pharmacological and functional properties, bind distinct epitopes on NCSTN ECD. This is currently being confirmed by peptide-based epitope mapping, as well as NCSTN deletion mutants (kind gift of Prof Christian Haass, Ludwig-Maximilians-University Munich, GE) $[2,18]$. In terms of translating our results collectively into a clinically applicable scenario, given that NCSTN is overexpressed in a subset of breast cancers, clinical stratification of patients eligible for NCSTN-targeted therapy could take the form of an RNAScope test for evaluation of target expression levels, while the $2 \mathrm{H} 6$ antibody can be considered for affinity maturation and humanisation for ultimate introduction into Phase I clinical trials. A companion diagnostic/ therapeutic approach like this has the potential to open up this challenging target beyond breast cancer therapy.

Acknowledgments We thank Cancer Research UK (CRUK), Breast Cancer Campaign (BCC) and Experimental Cancer Medicine Centre (ECMC) for supporting our work, Dr Knisely Alex, King's College Hospital NHS Trust, for his work on H\&E and PAS IHC for the mammary fat pad mouse model experiment, Dr Georgios Giamas and his group for their continuous support and intellectual and technical help, Pritesh Trivedi for his work on immunohistochemistry of excised mouse tumors and Advanced Cell Diagnostics (Hayward, CA, USA) for their expertise in performing the nicastrin RNAScope.

Conflict of interest None declared by all authors. Authors do not have a financial relationship with the organisation who sponsored the research.

Open Access This article is distributed under the terms of the Creative Commons Attribution Noncommercial License which permits any noncommercial use, distribution, and reproduction in any medium, provided the original author(s) and the source are credited.

\section{References}

1. Filipovic A, Gronau JH, Green AR, Wang J, Vallath S, Shao D, Rasul S, Ellis IO, Yague E, Sturge J et al (2011) Biological and clinical implications of nicastrin expression in invasive breast cancer. Breast Cancer Res Treat 125(1):43-53
2. Hayashi I, Takatori S, Urano Y, Miyake Y, Takagi J, SakataYanagimoto $\mathrm{M}$, Iwanari $\mathrm{H}$, Osawa $\mathrm{S}$, Morohashi $\mathrm{Y}$, Li $\mathrm{T}$ et al (2012) Neutralization of the gamma-secretase activity by monoclonal antibody against extracellular domain of nicastrin. Oncogene 31(6):787-798

3. Alattia JR, Schweizer C, Cacquevel M, Dimitrov M, Aeschbach L, Oulad-Abdelghani M, Fraering PC (2012) Generation of monoclonal antibody fragments binding the native gammasecretase complex for use in structural studies. Biochemistry 51(44):8779-8790

4. Yang DS, Tandon A, Chen F, Yu G, Yu H, Arawaka S, Hasegawa H, Duthie M, Schmidt SD, Ramabhadran TV et al (2002) Mature glycosylation and trafficking of nicastrin modulate its binding to presenilins. J Biol Chem 277(31):28135-28142

5. Kenny PA, Lee GY, Myers CA, Neve RM, Semeiks JR, Spellman PT, Lorenz K, Lee EH, Barcellos-Hoff MH, Petersen OW et al (2007) The morphologies of breast cancer cell lines in threedimensional assays correlate with their profiles of gene expression. Mol oncol 1(1):84-96

6. Lee GY, Kenny PA, Lee EH, Bissell MJ (2007) Three-dimensional culture models of normal and malignant breast epithelial cells. Nat Methods 4(4):359-365

7. Sahai E (2007) Illuminating the metastatic process. Nat Rev Cancer 7(10):737-749

8. Reymond N, Im JH, Garg R, Vega FM (2012) Borda d'Agua B, Riou P, Cox S, Valderrama F, Muschel RJ, Ridley AJ: Cdc42 promotes transendothelial migration of cancer cells through beta1 integrin. J cell biol 199(4):653-668

9. Fraering PC, Ye W, Strub JM, Dolios G, LaVoie MJ, Ostaszewski BL, van Dorsselaer A, Wang R, Selkoe DJ, Wolfe MS (2004) Purification and characterization of the human gammasecretase complex. Biochemistry 43(30):9774-9789

10. van Es JH, van Gijn ME, Riccio O, van den Born M, Vooijs M, Begthel H, Cozijnsen M, Robine S, Winton DJ, Radtke F et al (2005) Notch/gamma-secretase inhibition turns proliferative cells in intestinal crypts and adenomas into goblet cells. Nature 435(7044):959-963

11. Scully OJ, Bay BH, Yip G, Yu Y (2012) Breast cancer metastasis. Cancer Genom Proteomics 9(5):311-320

12. Gangadhara S, Barrett-Lee P, Nicholson RI, Hiscox S (2012) Prometastatic tumor-stroma interactions in breast cancer. Future oncol 8(11):1427-1442

13. Qamri Z, Preet A, Nasser MW, Bass CE, Leone G, Barsky SH, Ganju RK (2009) Synthetic cannabinoid receptor agonists inhibit tumor growth and metastasis of breast cancer. Mol Cancer Ther $8(11): 3117-3129$

14. Wang X, Liu J, Wang Z, Huang Y, Liu W, Zhu X, Cai Y, Fang X, Lin S, Yuan L et al (2013) Periostin contributes to the acquisition of multipotent stem cell-like properties in human mammary epithelial cells and breast cancer cells. PLoS ONE 8(8):e72962

15. Overall CM, Kleifeld O (2006) Tumour microenvironmentopinion: validating matrix metalloproteinases as drug targets and anti-targets for cancer therapy. Nat Rev Cancer 6(3):227-239

16. Cancer Genome Atlas (2012) N: Comprehensive molecular portraits of human breast tumours. Nature 490(7418):61-70

17. Wang F, Flanagan J, Su N, Wang LC, Bui S, Nielson A, Wu X, Vo HT, Ma XJ, Luo Y (2012) RNAscope: a novel in situ RNA analysis platform for formalin-fixed, paraffin-embedded tissues. J Mol Diagn 14(1):22-29

18. Zhang X, Hoey RJ, Lin G, Koide A, Leung B, Ahn K, Dolios G, Paduch M, Ikeuchi T, Wang R et al (2012) Identification of a tetratricopeptide repeat-like domain in the nicastrin subunit of gamma-secretase using synthetic antibodies. Proc Natl Acad Sci USA 109(22):8534-8539 